\title{
A NOTE ON THE ANGLES IN AN $n$-DIMENSIONAL SIMPLEX
}

\author{
by A. P. GUINAND
}

(Received 13th October, 1958)

1. Introduction. Three different sets of equations connecting the sums of angles in an $n$-dimensional simplex have been given by Sommerville [7], Höhn [5], and Peschl [6]. $\dagger$ The equivalence of the first two sets of equations has been proved by Sprott [7].

In the present note it is shown that results are simplified if we consider averages instead of sums, and that the averages form a sequence which is self-reciprocal with respect to the transformationt

$$
q_{s}=\sum_{r=0}^{s}(-1)^{r}\left(\begin{array}{l}
s \\
r
\end{array}\right) p_{r}
$$

The equivalence of the sets of equations is then easily proved by symbolic methods. $\$$

2. Forms of the equations. Given an $n$-dimensional simplex in spherical or Euclidean space, let $s_{k}$ denote the sum of the angles at its $\left(\begin{array}{c}n+1 \\ k\end{array}\right)(n-k)$-cells, each measured as a fraction of the whole angle at the $(n-k)$-flat concerned. Let $s_{0}=1$, and let $s_{n+1}$ be the content of the simplex as a fraction of the whole space.|l Then the three sets of equations are

$$
\begin{aligned}
& \sum_{k=r}^{n+1}(-1)^{k}\left(\begin{array}{l}
k \\
r
\end{array}\right) s_{k}=\sum_{k=n+1-r}^{n+1}(-1)^{n+1-k}\left(\begin{array}{c}
k \\
n+1-r
\end{array}\right) s_{k} \quad\left(0 \leqslant r \leqslant \frac{1}{2} n\right), \\
& \sum_{k=0}^{p}(-1)^{k}\left(\begin{array}{c}
n+1-k \\
n+1-p
\end{array}\right) s_{k}=s_{p} \quad\left(1 \leqslant p \leqslant 2\left[\frac{1}{2} n\right]+2\right), \\
& \sum_{k=1}^{l+1} \frac{2^{2 k}-1}{k}\left(\begin{array}{c}
n-2 l+2 k-1 \\
2 k-1
\end{array}\right) B_{2 k^{2}} s_{2 l-2 k+2}=s_{2 l+1} \quad\left(0 \leqslant l \leqslant \frac{1}{2} n\right),
\end{aligned}
$$

(Sommerville)

where $B_{2 k}$ runs through the Bernoulli numbers $B_{2}=\frac{1}{6}, B_{4}=-\frac{1}{30}, \ldots$

In each set there are only $\left[\frac{1}{2} n\right]+1$ independent equations. An independent set is obtained from Höhn's equations if we take the set of alternate values of $p$ which includes $p=n+1$.

Now put

$$
a_{k}=s_{k} /\left(\begin{array}{c}
n+1 \\
k
\end{array}\right)
$$

so that $a_{k}$ is the average angle at an $(n-k)$-cell, expressed as a fraction of the whole angle at an $(n-k)$-flat. In terms of the $a_{k}$ the equations become, respectively,

† See also Coxeter [2].

† Cf. Hardy [4].

$\S$ These results were suggested by similarities with equations arising in a problem about Fourier transforms. Cf. Guinand [3].

$\|$ That is, in the Euclidean case, $s_{n+1}=0$. Peschl [6] also shows that the same equations hold in hyperbolic space with an appropriate reinterpretation of $s_{n_{+1}}$. 


$$
\begin{gathered}
\sum_{k=r}^{n+1}(-1)^{k}\left(\begin{array}{c}
n+1-r \\
k-r
\end{array}\right) a_{k}=\sum_{k=0}^{r}(-1)^{k}\left(\begin{array}{l}
r \\
k
\end{array}\right) a_{n-k+1} \quad\left(0 \leqslant r \leqslant \frac{1}{2} n\right), \\
\sum_{k=0}^{r}(-1)^{k}\left(\begin{array}{l}
r \\
k
\end{array}\right) a_{k}=a_{r} \quad\left(1 \leqslant r \leqslant 2\left[\frac{1}{2} n\right]+2\right), \\
\sum_{k=0}^{r}\left(2^{r-k}-1\right)\left(\begin{array}{l}
r \\
k
\end{array}\right) B_{r-k} a_{k}=0 \quad(r \text { even, } 2 \leqslant r \leqslant n+2) .
\end{gathered}
$$

In these forms all three sets of equations can readily be expressed in the symbolic or umbral notation. If we put

$$
a^{k}=a_{k}, \quad B^{k}=B_{k},
$$

then the equations can be written, respectively,

$$
\begin{gathered}
a^{r}(1-a)^{n-r+1}=a^{n-r+1}(1-a)^{r} \quad\left(0 \leqslant r \leqslant \frac{1}{2} n\right), \\
(1-a)^{r}=a^{r} \quad\left(1 \leqslant r \leqslant 2\left[\frac{1}{2} n\right]+2\right), \\
(B+a)^{r}=(2 B+a)^{r} \quad(r \text { even, } 2 \leqslant r \leqslant n+2) .
\end{gathered}
$$

3. Equivalence of the sets of equations. Denote the sets of equations by $\mathbf{S}, \mathbf{H}, \mathbf{P}$ as above. Let $\mathrm{H}_{1}$ denote the set

and $\mathrm{H}_{2}$ the set

$$
(1-a)^{r}=a^{r} \quad(r \text { odd }, 1 \leqslant r \leqslant n+1)
$$

$$
(1-a)^{r}=a^{r} \quad(r \text { even, } 2 \leqslant r \leqslant n+2) .
$$

Then the equivalence of the four sets $\mathrm{S}, \mathrm{H}_{1}, \mathrm{H}_{2}, \mathrm{P}$ can be proved by the following stages.

(i) $\mathrm{H}_{1} \supset \mathrm{H}$. Suppose that $(1-a)^{r}=a^{r}$ for $1 \leqslant r \leqslant 2 q-1$. Then any symbolic polynomial in $a$ of degree not greater than $2 q-1$ is unchanged in value if $a$ is replaced by $1-a$. The polynomial $(1-a)^{2 q}-a^{2 q}$ is of degree $2 q-1$ only; hence it is equal to $a^{2 q}-(1-a)^{2 q}$, and therefore

$$
(1-a)^{2 q}=a^{2 q} \text {. }
$$

Now by $\mathrm{H}_{1}$ the result $(\mathrm{l}-a)^{r}=a^{r}$ is true for $r=1$; hence it is true for $r=1,2$. By $\mathrm{H}_{1}$ it is also true for $r=3$, and hence for $r=4$. Continuing this process, we see that it is true for $r=1,2,3, \ldots, 2\left[\frac{1}{2} n\right]+2$, as required.

(ii) $\mathrm{H}_{2} \supset \mathrm{H}$. Suppose that $(\mathrm{l}-a)^{r}=a^{r}$ for $0 \leqslant r \leqslant 2 q$ and also for $r=2 q+2$. Then the value of any symbolic polynomial in $a$ of degree not greater than $2 q$ is unchanged if $a$ is replaced by $1-a$. The polynomial

$$
(q+1)\left\{a^{2 q+1}-(1-a)^{2 q+1}\right\}-\left\{a^{2 q+2}-(1-a)^{2 q+2}\right\}
$$

is of degree $2 q$ only; hence, by an argument as in (i), it is equal to zero. Since $(1-a)^{2 q+2}=a^{2 q+2}$ by assumption, we have

$$
(1-a)^{2 q+1}=a^{2 q+1} .
$$

Now by $\mathrm{H}_{2}$ the result $(1-a)^{r}=a^{r}$ is true for $r=2$ and it is trivially true for $r=0$. Hence it is true for $r=1$. By $\mathrm{H}_{2}$ it is also true for $r=4$; so it is true for $r=0,1,2,4$, and and hence for $r=3$. Continuing the process, we see that it is true for $r=0,1,2, \ldots, 2\left[\frac{1}{2} n\right]+2$, as required. 
(iii) $\mathrm{H} \supset \mathrm{S}$. By $\mathrm{H}$ any symbolic polynomial in $a$ of degree not greater than $n+1$ is unchanged in value if $a$ is replaced by $1-a$. Hence

$$
a^{r}(1-a)^{n-r+1}=a^{n-r+1}(1-a)^{r}
$$

for $0 \leqslant r \leqslant n+1$. This includes $\mathrm{S}$.

(iv) $\mathrm{S} \supset \mathrm{H}$. The equations $\mathrm{S}$ run through the same set when $0 \leqslant r \leqslant \frac{1}{2} n$ and when $\frac{1}{2} n+1 \leqslant r \leqslant n+1$. If $n$ is odd, then the remaining equation with $r=\frac{1}{2} n+\frac{1}{2}$ is an identity. Hence $\mathrm{S}$ holds for $0 \leqslant r \leqslant n+1$. Thus for $0 \leqslant r \leqslant n$ we have both

and

$$
a^{r}(1-a)^{n-r+1}=a^{n-r+1}(1-a)^{r},
$$

$$
a^{r+1}(1-a)^{n-r}=a^{n-r}(1-a)^{r+1} .
$$

Adding these results, we have

$$
a^{r}(1-a)^{n-r}(1-a+a)=a^{n-r}(1-a)^{r}(a+1-a),
$$

or

$$
a^{r}(1-a)^{n-r}=a^{n-r}(1-a)^{r},
$$

for $0 \leqslant r \leqslant n$. Continuing this process, we get

$$
a^{p}(1-a)^{q}=a^{q}(1-a)^{p}
$$

for all $p$ and $q$ in $p \geqslant 0, q \geqslant 0, p+q \leqslant n+1$. On putting $q=0$ this gives $\mathrm{H}$, as required.

(v) $\mathrm{H} \supset \mathrm{P}$. The Bernoulli numbers are determined by the formal expansion

$$
e^{B x}=\sum_{m=0}^{\infty} B_{m} \frac{x^{m}}{m !}=\frac{x}{e^{x}-1}
$$

Hence the function $\phi(x)$, defined by the formal expansion

$$
\phi(x)=\sum_{m=0}^{\infty}\left\{(B+a)^{m}-(2 B+a)^{m}\right\} \frac{x^{m}}{m !},
$$

is equal to

$$
\begin{aligned}
e^{(B+a) x}-e^{(2 B+a) x} & =e^{a x}\left(e^{B x}-e^{2 B x}\right) \\
& =e^{a x}\left(\frac{x}{e^{x}-1}-\frac{2 x}{e^{2 x}-1}\right) \\
& =e^{(a-1) x}\left(\frac{1}{2} x \operatorname{sech} \frac{1}{2} x\right) \ldots
\end{aligned}
$$

Now if we replace $a$ by $1-a$ in $\left(a-\frac{1}{2}\right)^{r}$, it follows that $\mathrm{H}$ implies

for $r=0,1, \ldots, n+1$. Hence

$$
\left(a-\frac{1}{2}\right)^{r}=\left(\frac{1}{2}-a\right)^{r}
$$

$$
\left(a-\frac{1}{2}\right)^{r}=0
$$

for all odd $r$ not greater than $n+1$. Hence the expansion of $\phi(x)$ in the form (2) has no even powers of $x$ lower than $x^{n+2}$.

By (1) this implies that

$$
(B+a)^{r}-(2 B+a)^{r}=0
$$

for $r$ even and $0 \leqslant r \leqslant n+2$, as required. 
(vi) $\mathrm{P} \supset \mathrm{H}$. Reversing the argument of $(\mathrm{v})$, we see that $\mathrm{P}$ implies that $\left(a-\frac{1}{2}\right)^{r}=\left(\frac{1}{2}-a\right)^{r}$ for $0 \leqslant r \leqslant n+1$. Hence we can replace $\left(a-\frac{1}{2}\right)$ by $\left(\frac{1}{2}-a\right)$ in any polynomial in $a-\frac{1}{2}$ of degree not greater than $n+1$. Thus

$$
a^{r}=\left\{\frac{1}{2}+\left(a-\frac{1}{2}\right)\right\}^{r}=\left\{\frac{1}{2}+\left(\frac{1}{2}-a\right)\right\}^{r}=(1-a)^{r}
$$

for $0 \leqslant r \leqslant n+1$, as required.

(vii) $\mathrm{H}_{1} \equiv \mathrm{H}_{2} \equiv \mathrm{H} \equiv \mathrm{S} \equiv \mathrm{P}$. Since $\mathrm{H}$ includes $\mathrm{H}_{1}$ and $\mathrm{H}_{2}$, (i) and (ii) give $\mathrm{H}_{1} \equiv H \equiv \mathrm{H}_{2}$. Then (iii) and (iv) give $H \equiv S$, and (v) and (vi) give $S \equiv P$.

4. Remarks. If $\left\{p_{r}\right\}(r=0,1,2, \ldots)$ is any sequence, and $q_{s}$ is defined by

$$
q_{s}=\sum_{r=0}^{s}(-1)^{r}\left(\begin{array}{l}
s \\
r
\end{array}\right) p_{r} \quad(s=0,1,2, \ldots)
$$

then

$$
p_{s}=\sum_{r=0}^{s}(-1)^{r}\left(\begin{array}{l}
s \\
r
\end{array}\right) q_{r}
$$

Sequences connected by such a reciprocity may be called " reciprocal sequences" $\dagger$ With this terminology we can state the equations $H$ thus :

The sequence $\left\{a_{k}\right\}(k=0,1,2, \ldots, n+1)$ of angle averages at $(n-k)$-flats, expressed as fractions of the whole angle at an $(n-k)$-flat, is a self-reciprocal sequence.

A general solution of the equations $\mathrm{H}$ is given if we put $c^{r}=c_{r}$ where $\left\{c_{r}\right\}$ is any sequence. Then

or

$$
a^{r}=\left(\frac{1}{2}+c\right)^{r}+\left(\frac{1}{2}-c\right)^{r}
$$

is a general solution of $\mathrm{H}$.

$$
a_{r}=\sum_{k=0}^{[i+r]}\left(\begin{array}{c}
r \\
2 k
\end{array}\right)\left(\frac{1}{2}\right)^{2 k-1} c_{r-2 k}
$$

$\dagger$ Barrucand [1].

\section{REFERENCES}

1. P. Barrucand, Sur les suites reciproques, Comptes Rendus, 230 (1950), 1727-8.

2. H. S. M. Coxeter, Math. Reviews, 15 (1954), 55, and 18 (1957), 145.

3. A. P Guinand, Matrices associated with fractional Hankel and Fourier transforms, Proc. Glasgow Math. Assoc. 2 (1956), 185-92.

4. G. H. Hardy, Divergent series (Oxford, 1949), 247.

5. W. Höhn, Winkel and Winkelsumme im n-dimensionalen euklidischen Simplex, Thesis, Eidgenössische Technische Hochschule (Zürich, 1953).

6. E. Peschl, Winkelrelationen am Simplex und die Eulersche Charakteristik, Bayer. Akad. Wiss. Math.-Nat. Kl. S.-B., 1955, 319-45.

7. D. M. Y. Sommerville, The relations connecting the angle-sums and volume of a polytope in space of $n$ dimensions, Proc. Roy. Soc. London, A 115 (1927), 103-19.

8. D. A. Sprott, A combinatorial identity, Math. Gazette, 40 (1956), 207-9.

\section{UNIVERSITy OF ALbERTA}

\section{EDMONTON}

\section{Canada}

